\title{
STABILIZATION OF BILINEAR SYSTEMS WITH UNSTABLE DRIFT
}

\author{
MIGUEL TORRES-TORRITI AND HANNAH MICHALSKA \\ DEPARTMENT OF ELECTRICAL ENGINEERING, \\ MCGILL UNIVERSITY, \\ 3480 UNIVERSITY STREET, MONTRÉAL, P.Q. H3A 2A7 CANADA. \\ E-MAIL: MICHALSK@CIM.MCGILL.CA
}

\begin{abstract}
A simple and computationally feasible approach to feedback stabilization of a class of bilinear systems with unstable drift is presented. Feedback stabilization is carried out in two stages. In the first stage the system is steered to a stable manifold which corresponds to a special selection of constant controls in the original bilinear system. In this phase, a control Lyapunov function is used in conjunction with Lie algebraic control, allowing the system to reach a stable manifold in finite time. The Lie algebraic control involves a solution to a non-linear programming problem whose formulation results from a direct application of the Campbell-Baker-Hausdorff formula for composition of flows. In the second phase, the system is made to "slide" through the ensemble of stable manifolds corresponding to the largest set of constant controls for which such stable manifolds exist.
\end{abstract}

Keywords: Bilinear systems, stabilizing feedback, stable manifolds, Lie algebraic control.

\section{INTRODUCTION}

The aim of this paper is to present a method for the construction of discontinuous (in the state) stabilizing controls for a class of bilinear systems which are homogeneous in the state and defined on $\mathbb{R}^{n}$ :

$$
\dot{x}=A_{0} x+\sum_{k=1}^{m} u_{k} A_{k} x
$$

Here, $A_{k} \in \mathbb{R}^{n \times n}, k=0, \ldots m$, and $u_{k} \in \mathbb{R}$ are the control inputs, with $m<n$. Systems of this form are of practical relevance as they arise from the linearization of certain nonlinear systems with respect to the state only; see $[1,5]$. The stabilization problem for bilinear systems has thus received much attention in the literature, see for example $[1,2,3,4,5,6,7,8,9]$.

The research reported herein was sponsored by the NSERC of Canada under grant OGP-0138352. 
Unlike many existing stabilization methods, the control law proposed in this paper does not require the drift $A_{0} x$ to be critically stable, or stabilizable by constant controls. Our stabilization approach requires merely the existence of a constant control which renders the resulting linear system to have at least one eigenvalue in the open left half of the complex plane. By continuity, the latter induces the existence of a set of constant controls which yield a family of linear systems with stable manifolds. Our approach is thus suited for bilinear systems with inherently unstable drift terms. To our knowledge, feedback stabilization of such systems has not yet found a general solution (except for systems which evolve in the plane).

The proposed control law comprises two phases: the reaching phase and the sliding phase. In the reaching phase the state of the system is steered to a selected stable manifold by employing a suitably designed control Lyapunov function in conjunction with Lie algebraic control. The latter is necessary when there do not exist controls which generate instantaneous velocities decreasing the Lyapunov function. The Lie algebraic control is constructed in terms of a sequence of constant controls which yield a decrease in the value of the Lyapunov function after a finite time $T$. The control sequence is calculated as a solution to a non-linear programming problem whose formulation results from a direct application of the CampbellBaker-Hausdorff formula for composition of flows. Conditions are given under which the constructed feedback control renders the stable manifold globally attractive and attainable in finite time. Once the set of stable manifolds is reached the control is switched to its sliding phase whose task is to confine the motion of the closed loop system to the latter set, making it invariant under limited external disturbances.

The contributions of this paper can thus be summarized as follows:

- A novel Lie algebraic approach to the synthesis of stabilizing feedback control for homogeneous bilinear systems with an unstable drift is presented. The method applies to systems in which the drift cannot be stabilized by any constant control. Sufficient conditions for the existence of the proposed control law are given.

- Two examples corresponding to different dimension of the stable manifolds are presented to demonstrate the effectiveness of the approach.

\section{Problem Definition and Assumptions}

The objective in this paper is to present an approach to the synthesis of globally stabilizing feedback control for homogeneous bilinear systems of the type (1.1). For simplicity of exposition, we will explain 
our approach with reference to bilinear systems which, through the use of constant controls, yield linear systems with stable manifolds of co-dimension one with respect to the state space. Our approach can be generalized to systems with stable manifolds of any co-dimension, as is later shown in terms of an example.

For any set of constant controls $u \stackrel{\text { def }}{=}\left[u_{1}, \ldots, u_{m}\right]$, let

$$
A(u) \stackrel{\text { def }}{=} A_{0}+\sum_{k=1}^{m} u_{k} A_{k}
$$

The following basic assumptions will be needed:

A1. The controls $u_{k}, k=1, \ldots, m$ can vary freely over $\mathbb{R}$ and the feedback control law is sought in the class of piece-wise continuous functions in the state $x$ of the system.

A2. There exists constant controls $u^{*} \stackrel{\text { def }}{=}\left[u_{1}^{*}, \ldots, u_{m}^{*}\right]$ such that the corresponding linear system with system matrix $A\left(u^{*}\right)$ has $n-1$ stable eigenvalues $\lambda_{i}\left(u^{*}\right), i=1, \ldots, n-1$, i.e. $\lambda_{i}\left(u^{*}\right) \in \mathbb{C}^{-} \stackrel{\text { def }}{=}\{z \in$ $\mathbb{C} \mid \operatorname{Re}\{z\}<0\}, i=1, \ldots, n-1$.

A3. The system (1.1) satisfies the LARC condition for accessibility, namely that

$$
\operatorname{span} L\left\{A_{0} x, A_{1} x, \ldots, A_{m} x\right\}(x)=\mathbb{R}^{n}
$$

where $L\left\{A_{0} x, A_{1} x, \ldots, A_{m} x\right\}$ denotes the Lie algebra of vector fields $A_{0} x, A_{1} x, \ldots, A_{m} x$ and where

$$
L\left\{A_{0} x, A_{1} x, \ldots, A_{m} x\right\}(x)=\left\{z \in \mathbb{R}^{n} \mid z=A x, A x \in L\left\{A_{0} x, A_{1} x, \ldots, A_{m} x\right\}\right\}
$$

Additionally, (1.1) is completely controllable in the sense that for any two points $x_{0}, x_{f} \in \mathbb{R}^{n}$ there exists a piece-wise constant control which steers (1.1) from $x_{0}$ to $x_{f}$ in finite time.

Remark 2.1. Assumption A2 implies that there may not exist constant controls such that $A_{0}+\sum_{k=1}^{m} u_{k} A_{k}$ is stable.

\section{Stabilizing Feedback Control}

The underlying idea of the feedback synthesis is simple and draws on variable structure control approach. The feedback control comprises two stages: the reaching phase and the sliding phase. The task of the reaching phase control is to steer the system to a stable subspace in finite time, while the task of the sliding phase control is to keep the system's state evolving in the set of stable subspaces in the presence of limited external disturbances. 


\subsection{The reaching phase control.}

Let $\lambda_{k}\left(u^{*}\right)=a_{k}+i b_{k}, k=1, \ldots, n-1$ and let $w_{k}=s_{k}+i v_{k}, k=1, \ldots, n-1$ be the generalized eigenvectors of the matrix $A\left(u^{*}\right)$. Under assumption $\mathrm{A} 2$, let $n\left(u^{*}\right),\left\|n\left(u^{*}\right)\right\|=1$ be the normal vector to the stable and invariant subspace $E^{s}\left(u^{*}\right)=\operatorname{Span}\left\{s_{k}, v_{k} \mid a_{k}<0\right\}$. A generalized control Lyapunov function is defined by the signed distance to the stable subspace:

$$
V(x) \stackrel{\text { def }}{=} n^{T} x
$$

Along the trajectories of the system (1.1)

$$
\begin{array}{ll}
\frac{d V}{d t}(x) & \stackrel{\text { def }}{=} a(x)+b(x) u \\
\text { with } & a(x) \stackrel{\text { def }}{=} \nabla_{x} V A_{0} x, u \stackrel{\text { def }}{=}\left[u_{1}, \ldots, u_{m}\right] \\
& b(x) \stackrel{\text { def }}{=}\left[b_{1}(x) ; \ldots ; b_{m}(x)\right], b_{k}(x) \stackrel{\text { def }}{=} \nabla_{x} V A_{k} x, k=1, \ldots, m
\end{array}
$$

It is clear that the control

$$
u^{r}(x) \stackrel{\text { def }}{=} \frac{-a(x)-\eta \operatorname{sign}\{V(x)\}}{\|b(x)\|^{2}} b(x)^{T}
$$

with $\eta>0$ is well defined and bounded for all $x \notin \mathcal{N}_{\epsilon} E^{b}$ where $\mathcal{N}_{\epsilon} E^{b}$ denotes a suitably chosen $\epsilon$ neighbourhood of the set

$$
\begin{aligned}
E^{b} \quad & \stackrel{\text { def }}{=}\left\{x \in \mathbb{R}^{n} \mid b(x)=0\right\} \\
& =\left\{x \in \mathbb{R}^{n} \mid n^{T} A_{k} x=0, k=1, \ldots, m\right\}
\end{aligned}
$$

so that $\mathcal{N}_{\epsilon} E^{b} \stackrel{\text { def }}{=}\left\{x \in \mathbb{R}^{n} \mid\|b(x)\|<\epsilon\right\}$. Moreover, for all $x \notin \mathcal{N}_{\epsilon} E^{b}$

$$
\frac{d V}{d t}(x) \quad \stackrel{\text { def }}{=} a(x)+b(x) u^{r}(x)=-\eta \operatorname{sign}\{V(x)\}
$$

which implies that the distance between the current state of the system using the control $u^{r}$ and the stable subspace $E^{s}\left(u^{*}\right)$ is decreasing at a constant rate $\eta$ as long as the closed loop trajectory evolves in the complement of $\mathcal{N}_{\epsilon} E^{b}$.

Once the system trajectory enters $\mathcal{N}_{\epsilon} E^{b}$, a different control needs to be constructed as for $x \in \mathcal{N}_{\epsilon} E^{b}$ there may not exist any control value $u$ which renders $\frac{d V}{d t}(x)<0$ guaranteeing monotonic decrease in $V$. In this event, further (non-monotonic) decrease in the generalized Lyapunov function will be achieved as follows.

Consider a sequence of constant inputs $\left\{u_{(1)}, u_{(2)}, \ldots, u_{(s)}\right\}$ denoted by $\bar{u}$, and suppose that each $u_{(i)}$ is applied to $(1.1)$ for a time $\varepsilon_{i}, i=1, \ldots s$. Also, let $\bar{\varepsilon}$ denote the sequence of times $\left\{\varepsilon_{1}, \varepsilon_{2}, \ldots, \varepsilon_{s}\right\}$, and 
let $T=\sum_{i=1}^{s} \varepsilon_{i}$. Now, if $x(T ; x)$ denotes the state of (1.1) resulting from the application of $(\bar{u}, \bar{\varepsilon})$ to this system with initial condition $x$ at time $t=0$, then

$$
x(T ; x)=e^{\varepsilon_{s} A\left(u_{(s)}\right) x} \circ \cdots \circ e^{\varepsilon_{2} A\left(u_{(2)}\right) x} \circ e^{\varepsilon_{1} A\left(u_{(1)}\right) x} x
$$

where $e^{\varepsilon A(u) x} x$ denotes the solution of $\dot{x}=A(u) x$ through $x$ at $t=0$ evaluated at $t=\varepsilon$; i.e $e^{\varepsilon A(u) x}$ denotes the flow of $A(u) x$.

By virtue of the $\mathrm{CBH}$ formula, for sufficiently small times $\bar{\varepsilon}$, the composition of flows in (3.9) can be expressed in the form of a single flow; i.e. there exists a vector field $\bar{f}(x, \bar{u}, \bar{\varepsilon})$ such that

$$
x(T ; x)=e^{T \bar{f}(x, \bar{u}, \bar{\varepsilon})} x
$$

By assumption A3, the vector field $\bar{f}(x, \bar{u}, \bar{\varepsilon})$ has the following finite expression

$$
\bar{f}(x, \bar{u}, \bar{\varepsilon})=\sum_{i=1}^{N} c_{i}(\bar{u}, \bar{\varepsilon}) B_{i} x
$$

where $B_{i}, i=1, \ldots, N$, with $N \geq n$, is some basis for $L\left\{A_{0} x, A_{1} x, \ldots, A_{m} x\right\}$ and $c_{i}$ are coefficients which are nonlinear functions in the components of $(\bar{u}, \bar{\varepsilon})$, whose analytic expressions can be determined from the $\mathrm{CBH}$ formula, after collection of terms involving the same basis elements, see Example 2.

For a given $x \in \mathcal{N}_{\epsilon} E^{b}$ it is now possible to solve the following satisfycing problem with respect to the pair $(\bar{u}, \bar{\varepsilon})):$

Find a feasible pair $(\bar{u}, \bar{\varepsilon})$ such that :

$$
\operatorname{sign}\{V(x)\} n^{T} \sum_{i=0}^{N} c_{i}(\bar{u}, \bar{\varepsilon}) B_{i} x \leq-2 \eta
$$

where $\eta>0$ is some suitably chosen constant.

Let $\bar{u}(x, t), t \in[0, T]$ denote the concatenated control resulting from a solution pair $(\bar{u}, \bar{\varepsilon})$ to $(3.12)$; i.e. $\bar{u}(x, t)=u_{(k)}, t \in\left[t_{k}, t_{k}+\varepsilon_{k}\right], k=1, \ldots, s, t_{1}=0, t_{k}=t_{k-1}+\varepsilon_{k-1}, k=2, \ldots, s$.

The following partial results warrant the existence of solutions to the satisfycing problem (3.12) and establish its properties.

Proposition 1. Under assumption A3, for any $x \neq 0$, any time horizon $T>0$, and any constant $\eta>0$ there exists a solution to (3.12) provided that $s$, the number of switches in the control sequence $\bar{u}$, is allowed to be large enough, and that the CBH formula holds (globally) for all $(\bar{u}, \bar{\varepsilon})$. 
Proposition 2. Let assumption A3 hold and let $x(t ; x), t \in[0, T]$ denote the state of (1.1) resulting from the application of $\bar{u}(x, t)$ to this system at initial condition $x(0 ; x)=x$. There exists a time horizon $T>0$ such that for any $x \in \mathcal{N}_{\epsilon} E^{b}$

$$
\operatorname{sign}\{V(x)\}[V(x(T ; x))-V(x)]<-\eta T
$$

The proofs of these results can be found in [10].

The above discussion leads to the final definition of the reaching phase control and a subsequent characterization of its property.

Theorem 3.1. Suppose that assumption A3 holds. There exists a time horizon $T>0$ such that the reaching phase control defined by

$$
u^{R}(x, t) \stackrel{\text { def }}{=} \begin{cases}u^{r}(x) & x \notin \mathcal{N}_{\epsilon} E^{b} \\ \bar{u}(x, t) ; \text { for } t \in\left[t^{*}, t^{*}+T\right] & x \in \mathcal{N}_{\epsilon} E^{b}\end{cases}
$$

in which $t^{*}$ is the time instant at which $x$ is detected to be a member of the neighbourhood $\mathcal{N}_{\epsilon} E^{b}$, brings the system (1.1) to the stable subspace $E^{s}\left(u^{*}\right)$ in finite time.

The proof of this result can be found in [10].

\section{Remark 3.1.}

i. The reaching phase feedback control is time varying as the sequence of controls $\bar{u}(x, t)$ is applied (as an open loop control) over a time interval of length $T$ whenever the current state of the controlled system is detected to be a member of $\mathcal{N}_{\epsilon} E^{b}$

ii. The satisfycing problem is easier solved in two stages. To this end, an extended system to (1.1) can be defined first:

$$
\dot{x}=\sum_{k=1}^{N} v_{k} B_{k} x
$$

such that any instantaneous velocity of (3.15) can be achieved by a suitable selection of the "extended controls" $v_{k}, k=1, \ldots, N$. The latter is possible by virtue of Assumption A3. In the first stage, in intermediate solution to the satisfycing problem could then easily be found in terms of the extended controls by requiring that

$$
\left.\operatorname{sign}\{V(x)\} n^{T} \sum_{i=1}^{N} v_{i} B_{i} x\right\}<=-2 \eta
$$


A feasible solution to (3.12) could then be obtained by solving the least squares minimization

$$
\min _{(\bar{u}, \bar{\varepsilon})} \sum_{k=1}^{N}\left[v_{k}-c_{k}(\bar{u}, \bar{\varepsilon})\right]^{2}
$$

\subsection{The sliding phase control.}

Once the closed loop system using the control $u^{R}$ reaches the stable subspace $E^{s}\left(u^{*}\right)$ it is then logical to switch the control to the constant value $u^{*}$, which, in the absence of any external disturbances, keeps the system's state evolving in $E^{s}\left(u^{*}\right)$ for all future times. The latter results in asymptotic stabilization since $E^{s}$ is stable. As the assumption about the absence of external disturbances is not practical, a more realistic (feedback) version of the sliding control must take account of any possible deviations of the system's state from the desirable stable subspace $E^{s}\left(u^{*}\right)$. First let us notice the following consequence of continuity of the eigenvalues as functions of the control $u^{*}$, see [10].

Proposition 3. Let $u^{*} \in \mathbb{R}^{m}$ be a control which satisfies assumption A2. Then there exists a $\delta>0$ such that assumption $A 2$ holds for all $u \in \mathcal{U} \stackrel{\text { def }}{=} B\left(u^{*} ; \delta\right)$, where $B\left(u^{*} ; \delta\right) \subset \mathbb{R}^{m}$ is a ball of center $u^{*}$ and radius $\delta$. The mapping $u \mapsto n(u)$, with $n(u) \in \mathbb{R}^{n},\|n(u)\|=1$, the normal vector to $E^{s}(u)$, is continuous on $\mathcal{U}$. Consequently, the point to set mapping $u \mapsto E^{s}(u)$ is continuous on $\mathcal{U}$.

The sliding phase control is now defined on the set $\mathcal{S} \stackrel{\text { def }}{=} \bigcup_{u \in \mathcal{U}} E^{s}(u) \in \mathbb{R}^{n}$ :

$$
u^{S}(x) \stackrel{\text { def }}{=} u \text { for } x \in E^{s}(u)
$$

where, additionally, $u^{S}(x(t))$ is required to be continuous along any trajectory of the system $t \mapsto x(t) \in \mathcal{S}$. It is now possible to prove the following stabilization result, see [10].

Theorem 3.2. Suppose all assumptions A1-A3 are satisfied and let $T$ be such that the reaching control defined by (3.14) is steering the system from any initial point $x_{0} \in \mathbb{R}^{n} \backslash E^{s}\left(u^{*}\right)$ to the subspace $E^{s}\left(u^{*}\right)$ in finite time. Under these conditions, the combined reaching and sliding phase controls:

$$
u(x) \stackrel{\text { def }}{=} \begin{cases}u^{R}(x) & x \notin E^{s}\left(u^{*}\right) \\ u^{S}(x) & x \in \mathcal{S}\end{cases}
$$

provide an asymptotically stabilizing feedback control for system (1.1).

It should be noted that the regions of definition of the reaching and sliding controls are overlapping. In this context, definition (3.19) should be interpreted as one delivering a switching control $u$ with the 
switches occurring at time instants in which the closed loop system trajectory leaves any region currently traversed, be it $\mathcal{S}$ or $E^{s}\left(u^{*}\right)$.

\section{EXAMPLES}

4.1. Example 1: $\operatorname{dim} E^{s}\left(u^{*}\right)=2, n=3$.

The feedback control constructed is applied to a single-input bilinear system on $\mathbb{R}^{3}$, with the following matrices:

$$
A_{0}=\left[\begin{array}{rrr}
1 & -1 & 0 \\
1 & 1 & 0 \\
0 & 0 & 1
\end{array}\right] \quad A_{1}=\left[\begin{array}{rrr}
-1 & 0 & -1 \\
0 & 0 & 1 \\
1 & -1 & 1
\end{array}\right]
$$

It can easily be verified that the drift term $A_{0} x$ is unstable as all the eigenvalues of $A_{0}$ have positive real parts. It is also possible to verify that $A(u)=A_{0}+A_{1} u$ has at most two eigenvalues with negative real part for all $u$. The two stable eigenvalues of $A(u)$ occur for $u<0$ and are complex conjugate. It may also be verified that the Lie algebra generated by $A_{0} x$ and $A_{1} x$ satisfies the LARC condition.

The trajectories of the closed loop system are shown in Fig. 1.a for an initial state

$$
x_{0}=\left[\begin{array}{lll}
-0.0939 & 0.6460-0.7575
\end{array}\right]^{T}
$$

on $E^{b}$. The trajectory $x(t)$ never enters the set $E^{b}$ again, thus the control (3.6) is enough two reach $E^{s}$. A value of $u^{*}=-6$ was adopted for the design of the feedback $u^{r}(x)$, in which case

$$
n\left(u^{*}\right)=[-0.8860-0.3047-0.3496]^{T}
$$

In this simulation the constant rate of decrease $\eta$ was chosen to be 0.05 .

4.2. Example 2: $\operatorname{dim} E^{s}\left(u^{*}\right)=1, n=3$.

Consider a single-input bilinear system on $\mathbb{R}^{3}$ with matrices:

$$
A_{0}=\left[\begin{array}{rrr}
1 & -1 & 0 \\
1 & 1 & 0 \\
0 & 0 & -1
\end{array}\right] \quad A_{1}=\left[\begin{array}{rrr}
0 & -2 & 1 \\
2 & 0 & 1 \\
-1 & -1 & 0
\end{array}\right]
$$




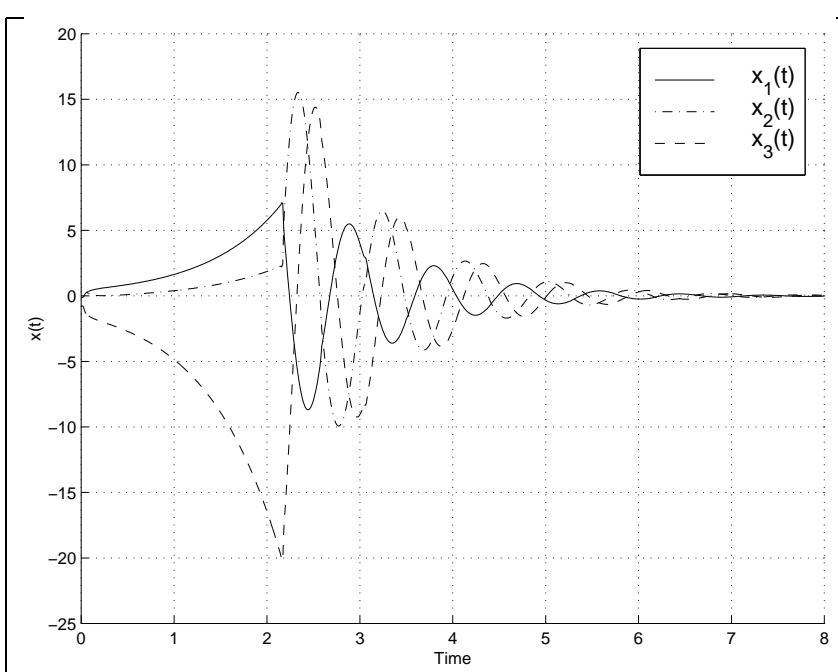

a. Close loop trajectory $x(t)$.

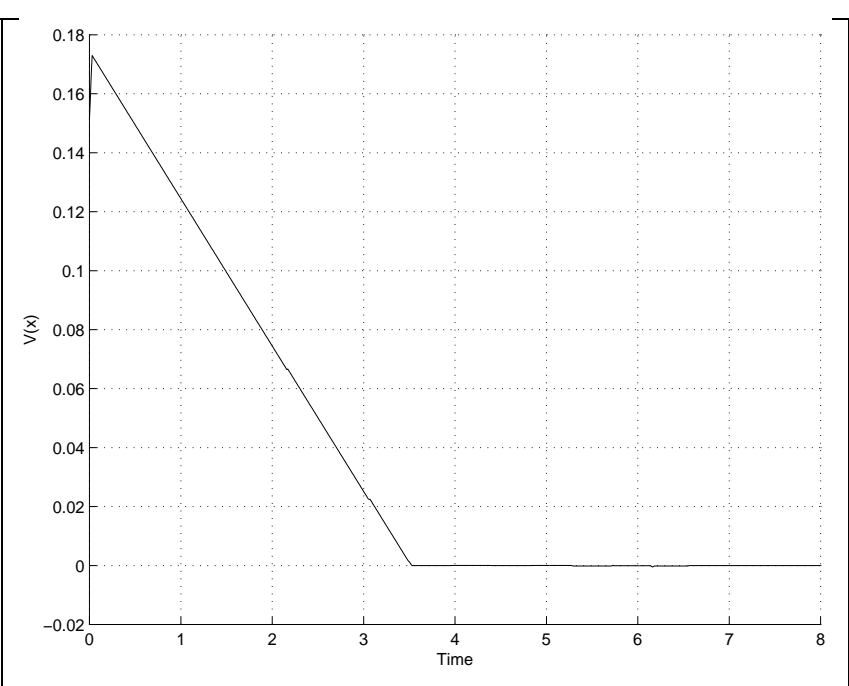

b. Lyapunov function $V(t)$.

FiguRE 1. Stabilization of system (4.20) to a stable plane, with initial condition $x_{0} \in E^{b}$.

In this example $A(u)=A_{0}+A_{1} u$ has only one stable eigenvalue regardless of the choice of $u \in \mathbb{R}$. The set $\mathcal{S}$ is then a collection of one dimensional subspaces $E^{s}$, where:

$$
E^{s}=\left\{x \in \mathbb{R}^{n} \mid x=s d(u), s \in \mathbb{R}\right\}
$$

Here $d(u)$ denotes the eigenvector corresponding to the stable eigenvalue $\lambda(u)$. It is now convenient to consider a control Lyapunov function $V(x)$ which is defined as the distance between the current state $x$ and its orthogonal projection on $E^{s}$, given by:

$$
V(x)=x^{T} x-\frac{\left(d^{T} x\right)^{2}}{d^{T} d}
$$

The reaching phase control $u^{r}(x)$ is hence defined as:

$$
u^{r}(x) \stackrel{\text { def }}{=} \frac{-a(x)-K}{\|b(x)\|^{2}} b(x)^{T}
$$

whenever $x \notin \mathcal{N}_{\epsilon} E^{b}$, with $K>0$ and

$$
E^{b}=\left\{x \in \mathbb{R}^{n} \mid x^{T}\left(d^{T} d A_{k}-d d^{T} A_{k}\right) x=0, k=1, \ldots, m\right\}
$$

It is easy to check that $E^{s} \subset E^{b}$, thus in the process of reaching $E^{s}$ it is inevitable for the state of the controlled system to enter the set $\mathcal{N}_{\epsilon} E^{b}$. This necessitates the construction of the time-varying component of the reaching control $(\bar{u}, \bar{\varepsilon})$. 
Let $X_{0}=A_{0} x$ and $X_{1}=A_{1} x$. The system satisfies the LARC, since

$$
\left.\operatorname{spanL}\left\{X_{0}, X_{1},\left[X_{0}, X_{1}\right],\left[X_{1},\left[X_{0}, X_{1}\right]\right]\right\}(x)\right)=\mathbb{R}^{3}
$$

The Lie algebra of this system is non-nilpotent, however it is finite dimensional and can be approximated by the following fourth order basis:

$$
\begin{aligned}
& B_{1}=X_{0} B_{2}=X_{1} \\
& B_{3}=\left[X_{0}, X_{1}\right] \quad B_{4}=\left[X_{0},\left[X_{0}, X_{1}\right]\right] \quad B_{5}=\left[X_{1},\left[X_{0}, X_{1}\right]\right] \\
& B_{6}=\left[X_{0},\left[X_{0},\left[X_{0}, X_{1}\right]\right]\right] \quad B_{7}=\left[X_{1},\left[X_{0},\left[X_{0}, X_{1}\right]\right]\right] \quad B_{8}=\left[X_{1},\left[X_{1},\left[X_{0}, X_{1}\right]\right]\right]
\end{aligned}
$$

The stable subspace $E^{s}$ is spanned by $d=\left[\begin{array}{lll}0 & 0 & 1\end{array}\right]^{T}$, which corresponds to the direction of the stable eigenvector generated with $u^{*}=0$ and for which

$$
V(x)=\frac{1}{2}\left(x_{1}^{2}+x_{2}^{2}\right)
$$

and the reaching phase feedback (3.14) may be written as:

$$
u^{R}(x)= \begin{cases}\frac{-\left(x_{1}^{2}+x_{2}^{2}\right)-K}{\left(x_{1}+x_{2}\right) x_{3}} & \text { for } x \notin \mathcal{N}_{\epsilon} E^{b} \\ \bar{u}\left(x\left(t^{*}\right), T\right) & \text { for } x \notin \mathcal{N}_{\epsilon} E^{b}\end{cases}
$$

with $\mathcal{N}_{\epsilon} E^{b}=\left\{x \mid\left(x_{1}+x_{2}\right) x_{3}<\epsilon\right\}, \epsilon=0.01$.

The computation of the values the control sequence $\bar{u}$ requires of the expressions for the coefficients $c_{i}(\bar{u}, \bar{\varepsilon})$ that define the vector field $\bar{f}(x, \bar{u}, \bar{\varepsilon})$ of equation $(3.11)$, resulting from the composition of flows in 
equation (3.9). Considering a control sequence $\bar{u}$ with $s=4$, application of the CBH formula yields:

$$
\begin{aligned}
c_{1}(\bar{u}, \bar{\varepsilon})= & T \\
c_{2}(\bar{u}, \bar{\varepsilon})= & \left(u_{(1)}+u_{(2)}+u_{(3)}+u_{(4)}\right) \epsilon \\
c_{3}(\bar{u}, \bar{\varepsilon})= & \left(-3 u_{(1)}-u_{(2)}+u_{(3)}+3 u_{(4)}\right) \frac{\epsilon^{2}}{2} \\
c_{4}(\bar{u}, \bar{\varepsilon})= & \left(u_{(1)}-u_{(2)}-u_{(3)}+u_{(4)}\right) \frac{\epsilon^{3}}{2} \\
c_{5}(\bar{u}, \bar{\varepsilon})= & \left(-3 u_{(1)}^{2}-2 u_{(1)} u_{(2)}+4 u_{(1)} u_{(3)}-3 u_{(2)}^{2}-2 u_{(3)} u_{(2)}\right. \\
& \left.-3 u_{(3)}^{2}+10 u_{(4)} u_{(1)}+4 u_{(4)} u_{(2)}-2 u_{(4)} u_{(3)}-3 u_{(4)}^{2}\right) \frac{\epsilon^{3}}{12} \\
c_{6}(\bar{u}, \bar{\varepsilon})= & \left(5 u_{(1)}+7 u_{(2)}-3 u_{(3)}-9 u_{(4)}\right) \frac{\epsilon^{4}}{24} \\
c_{7}(\bar{u}, \bar{\varepsilon})= & \left(5 u_{(1)}^{2}+4 u_{(1)} u_{(2)}+u_{(3)}^{2}-4 u_{(4)} u_{(3)}+4 u_{(4)} u_{(2)}-9 u_{(4)}^{2}-4 u_{(1)} u_{(3)}+3 u_{(2)}^{2}\right) \frac{\epsilon^{4}}{24} \\
c_{8}(\bar{u}, \bar{\varepsilon})= & \left(-5 u_{(4)}^{2} u_{(1)}-3 u_{(4)}^{2} u_{(2)}-u_{(4)}^{2} u_{(3)}+5 u_{(4)} u_{(1)}^{2}\right. \\
& \left.+4 u_{(4)} u_{(1)} u_{(2)}-4 u_{(4)} u_{(1)} u_{(3)}+3 u_{(4)} u_{(2)}^{2}+u_{(4)} u_{(3)}^{2}\right) \frac{\epsilon^{4}}{24}
\end{aligned}
$$

for $\varepsilon_{k}=\epsilon, k=1,2,3,4$, with $\epsilon=T / 4$ and the basis (4.27).

With the above $c_{i}$ the control $\bar{u}$ is computed by solving (3.12) via nonlinear least squares procedures.

The stabilization of (4.21), by means of (4.28) and the sliding phase control with $u^{*}=0$ is shown in Fig. 2. Note that the initial condition, $x_{0}=\left[\begin{array}{llll}-0.1 & 0.1 & 0.4\end{array}\right]^{T}$, lies in $E^{b}$. The control sequence period is selected to be $T=1$. The simulation results show that the trajectory $x(t)$ stays in $\mathcal{N}_{\epsilon} E^{b}$ for almost the first 0.5 seconds until it enters $\mathcal{S}$.

\section{Conclusions}

Switching stabilizing control of bilinear systems has previously been proposed only for non-homogeneous bilinear systems [2], or for single input systems with a dyadic $A_{1}$ matrix [3].

The contribution of this paper is a novel approach to the synthesis of stabilizing feedback control for homogeneous bilinear systems with an unstable drift. The method applies to systems in which the drift cannot be stabilized by any constant control. The examples presented demonstrate the effectiveness of the approach. 


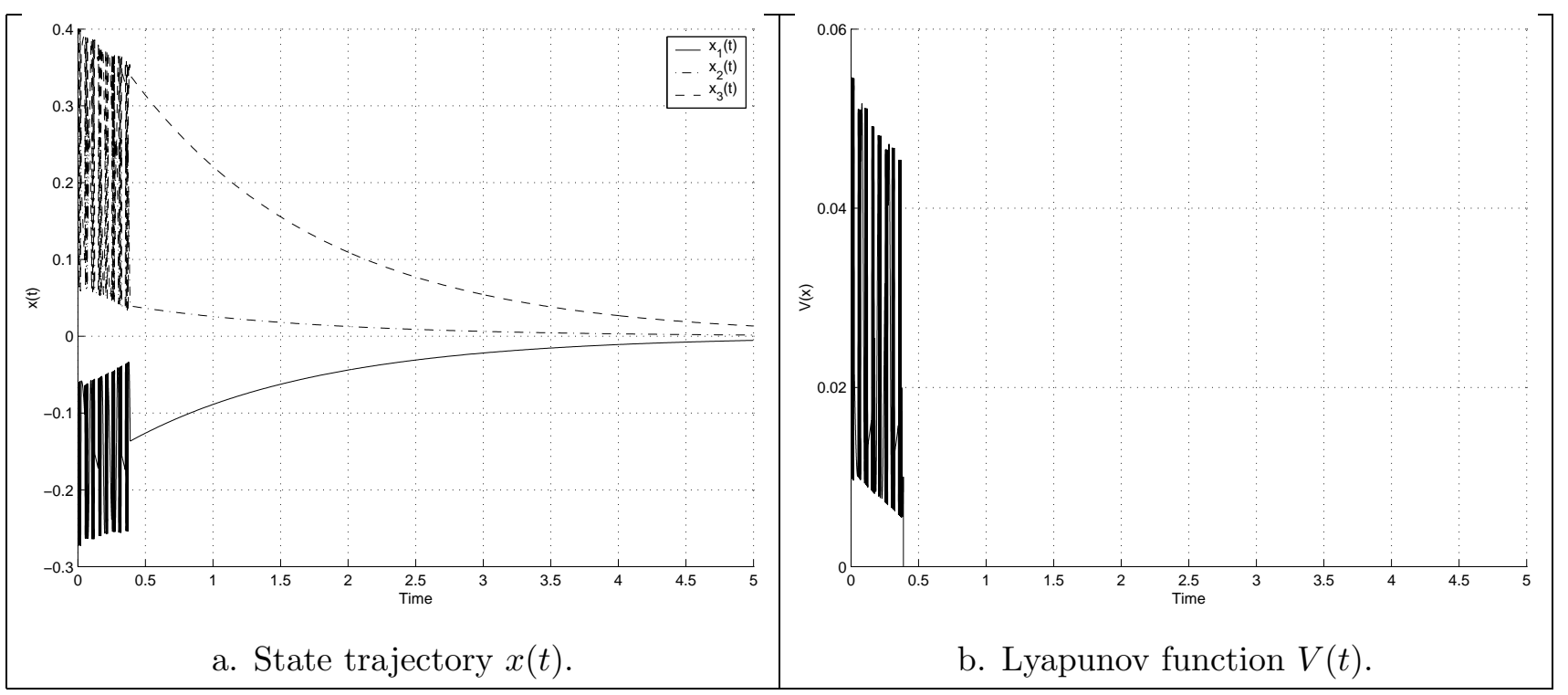

FiguRE 2. Stabilization system (4.21) to the stable line, with initial condition $x_{0} \in E^{b}$.

\section{REFERENCES}

[1] D. L. Elliott, "Bilinear Systems", in Encyclopedia of Electrical Engineering, edited by John Webster, John Wiley and Sons, 1999.

[2] R. Longchamp, "Stable Feedback Control of Bilinear Systems", IEEE Transactions on Automatic Control, Vol. AC-25, No. 2, pp. 302-306, April 1980.

[3] Per-Olof Gutman, "Stabilizing Controllers for Bilinear Systems", IEEE Transactions on Automatic Control, Vol. AC-26, No. 4, pp. 917-922, August 1981.

[4] S. Čelikovský, "On the Stabilization of the Homogeneous Bilinear Systems", Systems ES Control Letters, No. 21, pp. 503-510, North-Holland, 1993.

[5] R. Chabour, G. Sallet, J. C. Vivalda, "Stabilization of Nonlinear Systems: A Bilinear Approach", Mathematics of Control, Signals and Systems, No. 6, pp. 224-246, 1993.

[6] H. Wang, "Feedback Stabilization of Bilinear Control Systems", SIAM Journal on Control and Optimization, Vol. 36, No. 5, pp. 1669-1684, September 1998.

[7] M.-S. Chen, "Exponential Stabilization of a Constrained Bilinear System", Automatica, Vol. 34, No. 8, pp. 989-992, 1998.

[8] G. Lu, Z. Yufan, Z. Cishen, Y. Xinghuo, "Global Asymptotic Stabilization of MIMO Bilinear Systems with Undamped Natural Response", Proceedings of the 37th Conference on Decision and Control, pp.1989-1994, Tampa, Florida, December 1998.

[9] H. Michalska, M. Torres-Torriti, "Time Varying Stabilising Feedback Design for Bilinear Systems", Proceedings of the IASTED International Conference on Modelling, Identification and Control - MIC'2000, Innsbruck, Austria, February 2000 . 
[10] H. Michalska, M. Torres-Torriti, "Stabilization of Bilinear Systems with Unstable Drift", Internal Report, Department of Electrical and Computer Engineering, McGill University, 2001. 